Internist 2021 - 62:572-576

https://doi.org/10.1007/s00108-021-01022-z

Angenommen: 16. März 2021

Online publiziert: 13. April 2021

(c) Springer Medizin Verlag GmbH, ein Teil von

Springer Nature 2021

\section{Redaktion}

C. Bokemeyer, Hamburg

M. Hallek, Köln

C. Jacobshagen, Karlsruhe

W. Lehmacher, Köln

U. Müller-Ladner, Bad Nauheim

H. Wedemeyer, Hannover

M. Wehling, Mannheim

\section{Originalliteratur}

Chen P, Nirula A, Heller B et al for the BLAZE1-Investigators (2021) SARS-CoV-2 neutralizing antibody LY-CoV555 in outpatients with Covid-19. N Engl J Med 384:229-237. https:// doi.org/10.1056/NEJMoa2029849

Weinreich DM, Sivapalasingam S, Nortona T et al (2021) REGN-COV2, a neutralizing antibody cocktail, in outpatients with Covid-19. N Engl J Med 384:238-251. https://doi.org/10. 1056/NEJMoa2035002

Seit der ersten beschriebenen Infektion mit dem „severe acute respiratory syndrome coronavirus 2" (SARS-CoV-2) im Dezember 2019 hat sich die „coronavirus disease 2019“ (COVID-19) zu einer Pandemie entwickelt, deren Symptomatik von einem asymptomatischen Verlauf bis hin zu akutem Lungen- und Multiorganversagen sowie Tod reicht. Unterschiedliche medikamentöse Therapieansätze wurden verfolgt, so etwa die Therapie mit Remdesivir [1] oder Dexamethason, eine spezifische Therapie gibt es bisher jedoch nicht. In präklinischen Studien konnte eine Reduktion der Viruslast und damit ein leichterer Erkrankungsverlauf durch die Gabe spezifischer Antikörper erreicht werden. Die Food and Drug Administration (FDA) hat in den USA bereits für beide nachfolgend vorgestellten Antikörper eine Notfallzulassung auf Basis der Ergebnisse von PhaseII-Studien erteilt.

Ziel der im Folgenden diskutierten Studien war es, die Wirksamkeit einer

\author{
M. Augustin ${ }^{1} \cdot$ M. Hallek' $\cdot$ S. Nitschmann ${ }^{2}$ \\ ' Klinik I für Innere Medizin, Universitätsklinikum Köln (AöR), Köln, Deutschland \\ ${ }^{2}$ Lippetal, Deutschland
}

\title{
Antikörpertherapie bei Patienten mit COVID-19
}

monoklonalen Antikörpertherapie bei ambulanten Patienten mit COVID-19Infektion zu verifizieren.

\section{Zusammenfassung der Studien}

\section{S. Nitschmann \\ Lippetal, Deutschland}

\section{BLAZE-1: neutralisierender Antikörper LY-CoV5551}

\section{Studiendesign}

Laufende doppelblinde, placebokontrollierte, randomisierte 4 -armige $(700 \mathrm{mg}$ vs. $2800 \mathrm{mg}$ vs. $7000 \mathrm{mg}$ vs. Placebo) Multicenterstudie an 41 Zentren in den USA mit LY-CoV555 (synonym LY3819253; Bamlanivimab), einem monoklonalen neutralisierenden Antikörper („neutralizing antibody" $[\mathrm{NAb}]$ ) gegen das SpikeProtein, der mit hoher Affinität an die Rezeptorbindungsdomäne von SARSCoV-2 bindet, mit einer 29-tägigen Nachuntersuchungszeit

\footnotetext{
1 In der Phase-II-Studie BLAZE-2 wurde Bamlanivimab bei Bewohnern und Betreuern von Seniorenheimen präventiv eingesetzt. Laut Hersteller sank das Erkrankungsrisiko hierdurch um $80 \%$. Die Publikation der Studienergebnisse steht noch aus (https://www.vfa.de/de/ arzneimittel-forschung/woran-wir-forschen/ therapeutische-medikamente-gegen-diecoronavirusinfektion-covid-19).
}

\section{Einschlusskriterien}

- Ambulante Patienten mit positivem SARS-CoV-2-Nachweis und milden bis moderaten COVID-19Symptomen

\section{Endpunkte \\ Primär:}

- Änderung der SARS-CoV-2-Viruslast an Tag 11 ( \pm 4 Tage) gegenüber dem Ausgangswert

Sekundär:

- Sicherheit

- Symptomatik (Fragebogen)

- Klinik: kombiniert aus COVID-19assoziierter stationärer Aufnahme, Vorstellung in der Notaufnahme und Tod

- Änderung der SARS-CoV-2-Viruslast an Tag 3 und Tag 7

Take home message

Zusammengefasst konnten im Vergleich zur Placebogruppe die frühzeitig intravenös verabreichten neutralisierenden Antikörperkonzentrate LY-CoV555 und REGNCOV2 zur stärkeren Senkung der SARS-CoV-2Viruslast im Nasenrachenraum führen sowie Hospitalisierungen bzw. Arztkontakte von ambulanten COVID-19-Patienten verringern. Ob die Senkung der Ausgangsviruslast auch direkte klinische "benefits" in Bezug auf COVID-19-assoziierte Symptome hat, sollte in weiterführenden Studien untersucht werden. 


\begin{tabular}{|c|c|c|c|}
\hline Dosierung & LY-CoV555 & Placebo & Differenz (95\%-Konfidenzintervall) \\
\hline \multicolumn{4}{|l|}{ Primärer Endpunkt } \\
\hline Änderung der SARS-CoV-2-Viruslast an Tag 11 & - & $-3,47$ & - \\
\hline $700 \mathrm{mg}$ & $-3,67$ & - & $-0,20(-0,66$ bis 0,25$)$ \\
\hline $2800 \mathrm{mg}$ & $-4,00$ & - & $-0,53(-0,98$ bis $-0,08)$ \\
\hline $7000 \mathrm{mg}$ & $-3,38$ & - & $0,09(-0,37$ bis 0,55$)$ \\
\hline Gepoolte Dosis & $-3,70$ & - & $-0,22(-0,60$ bis 0,15$)$ \\
\hline \multicolumn{4}{|l|}{ Sekundäre Endpunkte } \\
\hline Änderung der SARS-CoV-2-Viruslast an Tag 3 & -1,35 (gepoolte Dosis) & $-0,85$ & $-0,49(-0,87$ bis $-0,11)$ \\
\hline Änderung der SARS-CoV-2-Viruslast an Tag 7 & $-2,90$ (gepoolte Dosis) & $-2,56$ & $-0,33(-0,72$ bis 0,06$)$ \\
\hline
\end{tabular}

\section{Methodik}

Die 467 Patienten für diese Interimsanalyse wurden zwischen dem 17.06. und 12.08.2020 randomisiert. Nach Studieneinschluss wurde den Patienten binnen 3 Tagen nach dem positiven SARSCoV-2-Nachweis die Studienmedikation als einmalige einstündige Infusion verabreicht:

- 101 Patienten erhielten 700 mg LYCoV555,

- 107 Patienten erhielten 2800 mg LYCoV555,

- 101 Patienten erhielten 7000 mg LYCoV555,

- 143 Patienten erhielten Placebo.

Der Nachweis der Viruslast erfolgte mittels quantitativer Reverse-TranskriptasePolymerase-Kettenreaktion (RT-PCR) eines Nasopharyngealabstrichs.

\section{Ergebnisse}

Es werden die Daten einer geplanten Interimsanalyse der noch laufenden Multicenterstudie vorgestellt.

Das durchschnittliche Patientenalter betrug 45 Jahre, wobei $12 \%$ der Patienten $\geq 65$ Jahre alt waren. Insgesamt waren $55 \%$ der Patienten Frauen und beinahe $70 \%$ der Patienten wiesen einen Risikofaktor (Alter $\geq 65$ Jahre, Body-Mass-In$\operatorname{dex}[\mathrm{BMI}] \geq 35 \mathrm{~kg} / \mathrm{m}^{2}$ oder eine relevante Begleiterkrankung) für einen schweren Krankheitsverlauf auf. Bei Randomisierung litten $80 \%$ der Patienten unter milden Symptomen, die seit durchschnittlich 4 Tagen bestanden. Die durchschnittliche Zyklusschwelle der RT-PCR entsprach 23,9 Ct (Ct „cycle threshold“; geringe- rer Ct-Wert $=$ höhere Viruslast $=$ höhere Infektiosität).

Bis Tag 11 sank die Viruslast in allen vier Gruppen, im Durchschnitt um -3,81, was mit einer Abnahme um den Faktor 4300, entsprechend einer Reduktion der viralen RNA um 99,97\%, gleichzusetzen ist. Die Abnahme der Viruslast war bei den Patienten der 2800 mg-Gruppe verglichen mit den Patienten der Placebogruppe am besten ( 0 Tab. 1).

In der gepoolten Studienpopulation konnte eine Korrelation zwischen langsamer Viruslastabnahme und Häufigkeit der Krankenhausaufnahme gezeigt werden. An Tag 7 war die Viruslast der hospitalisierten Patienten höher als die der ambulanten Patienten und von den $\mathrm{Pa}$ tienten mit einem Ct-Wert $<27,5$ waren $12 \%$ hospitalisiert, verglichen mit 0,9\% bei den Patienten mit höherem Ct-Wert.

An Tag 29 waren 1,6\% der LYCoV555- und 6,3\% der Placebopatienten hospitalisiert; bei Patienten $\geq 65$ Jahre oder mit einem $\mathrm{BMI} \geq 35 \mathrm{~kg} / \mathrm{m}^{2}$ waren dies $4 \%$ bzw. $15 \%$. Lediglich ein Placebopatient wurde intensivpflichtig. Die subjektive Symptomatik wurde von den LY-CoV555-Patienten besser angegeben als von den Placebopatienten.

Schwere Nebenwirkungen traten bei keinem der LY-CoV555- und bei einem der Placebopatienten auf. Insgesamt kam es bei 22,3\% der LY-CoV555- und bei $24,5 \%$ der Placebopatienten zu Nebenwirkungen, wobei Übelkeit und Diarrhö am häufigsten auftraten.

\section{Weinreich et al.: REGN-COV2²}

\section{Studiendesign}

Laufende doppelblinde, placebokontrollierte, randomisierte 3 -armige $(2,4 \mathrm{~g}$ vs. $8 \mathrm{~g}$ vs. Placebo) Multicenterstudie mit REGN-COV2, einem „Cocktail“ aus zwei nichtkonkurrierenden, humanen Immunglobulin-G-Typ-1(IgG1)-Antikörpern (Casirivimab und Imdevimab im Verhältnis 1:1), die beide mit hoher Affinität an die Rezeptorbindungsdomäne von SARS-CoV-2 binden

\section{Einschlusskriterien}

- Symptomatische COVID-19-positive Erwachsene in ambulantem Setting

- Symptombeginn nicht mehr als

7 Tage vor Randomisierung

- Positiver COVID-19-Nachweis nicht mehr als $72 \mathrm{~h}$ vor Randomisierung

\section{Endpunkte}

Primär:

- Änderung der Viruslast von Tag 1 bis Tag 7 mittels RT-PCR eines Nasopharyngealabstrichs in $\log _{10}$ Kopien/ml bei Serumantikörper-negativen Patienten

- Anzahl der Patienten, die sich bis zum Tag 29 aufgrund der COVID19-Symptomatik in medizinische Behandlung begeben hatten

\section{Sekundär:}

\section{- Sicherheit}

\footnotetext{
2 Das „Rolling-review-Verfahren“ bei der Europäischen Arzneimittel-Agentur (EMA) ist initiiert.
} 


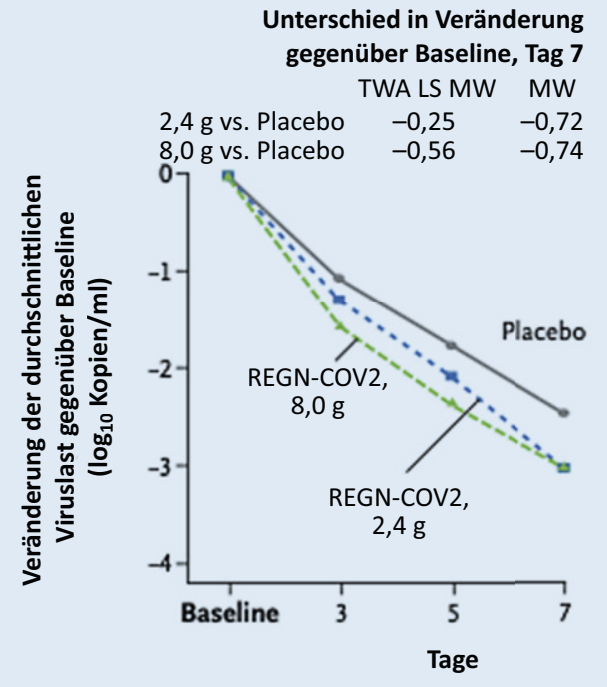

Anzahl unter Risiko

Placebo

REGN-COV2, 2,4 g

REGN-COV2, 8,0 g

$\begin{array}{llll}81 & 70 & 78 & 78 \\ 73 & 66 & 69 & 70 \\ 74 & 70 & 73 & 73\end{array}$

Abb. $1<$ Viruslast über die Zeit: durchschnittliche Veränderung in $\log _{10}$ Kopien $/ \mathrm{ml} . L S$ "least squares" (kleinste Quadrate), MW Mittelwert, TWA „timeweighted mean" (zeitgewichteter Durchschnitt). (Adaptiert nach Abb. 2 a von S. 248 der Originalpublikation Weinreich et al)

\section{Methodik}

Die 275 Patienten für diese Interimsanalyse wurden zwischen dem 16.06 . und dem 18.08.2020 randomisiert. Nach Studieneinschluss wurde den Patienten binnen $72 \mathrm{~h}$ nach dem positiven SARSCoV-2-Nachweis die Studienmedikation als einmalige einstündige Infusion verabreicht:

- 92 Patienten sollten 2,4g REGN-

COV2 (LD) erhalten, von denen 80

die Studie beendeten.

- 90 Patienten sollten 8,0 g REGN-

COV2 (HD) erhalten, von denen 84 die Studie beendeten.

- 93 Patienten sollten Placebo erhalten, von denen 88 die Studie beendeten.

Der Nachweis der Viruslast erfolgte mittels quantitativer RT-PCR eines Nasopharyngealabstrichs.

$\mathrm{Zu}$ Studienbeginn wurde bei allen $\mathrm{Pa}$ tienten ein Anti-SARS-CoV-2-Antikörper-Nachweis initiiert.

\section{Ergebnisse}

Es werden die Daten einer geplanten Interimsanalyse nach den ersten 275 rekrutierten Patienten der noch laufenden Multicenterstudie vorgestellt.

Das durchschnittliche Patientenalter betrug 44 Jahre, 51\% waren Frauen und der durchschnittliche BMI betrug
$30 \mathrm{~kg} / \mathrm{m}^{2}$. Bei Randomisierung betrug die mediane Viruslast 156.000 Kopien/ml (Range 1-71.000.000 Kopien/ml), die durchschnittliche Viruslast lag bei 15.970.000 Kopien/ml. Ein positiver RTPCR-Test lag bei $83 \%$ der Patienten vor. Der durchschnittliche Wert des C-reaktiven Proteins betrug 15,1 g/l, der mediane Wert 4,1 g/l (Range 0,1-239,7 g/l). Bei Randomisierung konnten bei $45 \%$ der Patienten Antikörper nachgewiesen werden.

Die Viruslast von Tag 1 bis 7 bei Serumantikörper-negativen Patienten war bei den LD-REGN-COV2-Patienten um $0,52 \log _{10}$ Kopien/ml geringer als bei den Placebopatienten (95\%-Konfidenzintervall $-1,04$ bis $0,00 \log _{10} \mathrm{Kopien} / \mathrm{ml}$ ); bei den HD-REGN-COV2-Patienten um $0,60 \log _{10}$ Kopien/ml geringer als bei den Placebopatienten (95\%-Konfidenzintervall $-1,12$ bis $-0,08 \log _{10}$ Kopien/ml). In der gepoolten REGN-COV2-Gruppe war die Viruslast um 0,56 $\log _{10}$ Kopien/ml geringer (95\%-Konfidenzintervall -1,02 bis -0,11 $\log _{10}$ Kopien/ml; • Abb. 1).

Es konnte gezeigt werden, dass die Patienten mit der höchsten Viruslast am meisten von der Antikörpertherapie profitierten: Die Viruslast sank bei Patienten, deren Ausgangsviruslast mehr als $10^{7}$ Kopien/ml betrug, bis Tag 7 durch die REGN-COV2-Therapie 2-mal mehr als bei den Placebopatienten, wobei die maximale Viruslastreduktion an Tag 3 nachgewiesen werden konnte.

Medizinische Hilfe nahmen 6 der 93 Placebo- und 6 der 182 REGNCOV2-Patienten in Anspruch (6\% vs. $3 \%$ ); bei den Serumantikörper-negativen Patienten waren dies 5 von 33 Placebound 5 von 80 REGN-COV2-Patienten (15\% vs. $6 \%)$.

Es traten nur geringe Therapienebenwirkungen bei $2 \%$ der Placebo- und $1 \%$ der REGN-COV2-Patienten auf.

\section{Kommentar}

Dr. med. M. Augustin, Prof. Dr. M. Hallek

Klinik I für Innere Medizin, Universitätsklinikum Köln (AöR), Köln, Deutschland

Die neutralisierende Antikörpertherapie fällt unter das Prinzip der passiven Immunisierung. Hier werden bereits fertige, meist humane Antikörperkonzentrate verabreicht, die sofort ihre Funktionen im Körper übernehmen können. Nach etwa 8-16 Wochen werden jene Antikörper vorrangig in der Milz und in den Zellen des mononukleär-phagozytären Systems abgebaut. Im Gegensatz dazu steht die aktive Immunisierung, bei der antigenvermittelt - beispielsweise über das Spike-Protein bei den Impfungen zur Prävention von COVID-19 - das eigene Immunsystem zur Antikörperproduktion angeregt wird.

Die hier vorliegenden Zwischenanalysen von randomisierten, doppelblinden und placebokontrollierten Studien bewerten die Sicherheit und Wirksamkeit der intravenös verabreichten SARSCoV-2-NAb LY-CoV555 (Eli Lilly, Indianapolis, Indiana, USA; einzelner NAb: Bamlanivimab) und REGN-COV2 (Regeneron, Tarrytown, New York, USA; Cocktail aus zwei nichtkonkurrierenden NAb: Casirivimab und Imdevimab) an ambulanten COVID-19-Patienten mit milden Symptomen (Score von 1 bis 3 auf der World Health Organization [WHO] Clinical Progression Scale [2]). Der primäre, virologische Endpunkt in beiden Studien war die Veränderung der SARS-CoV-2-Viruslast an Tag 11 bzw. 7. Weitere klinische Endpunkte untersuchten die Rate an Hospitalisierungen bis Tag 29 bzw. die Anzahl der 
medizinischen Behandlungen bis Tag 29 sowie in beiden Studien die Sicherheit. Die Studienpopulationen waren in Bezug auf medianes Alter, Geschlecht, BMI sowie Ethnien vergleichbar. Die Verabreichung von Verum bzw. Placebo erfolgte in beiden Studien in verschiedenen Dosisstufen (niedrige Dosis [LD]; mittlere Dosis [MD]; hohe Dosis [HD]) und im frühen Krankheitsstadium 4 bzw. 3 Tage nach Symptombeginn.

Bei genauerer Betrachtung der virologischen Endpunkte führte sowohl LY-CoV555 als auch REGN-COV2 im Vergleich zur Placebogruppe zu einem größeren Abfall der Ausgangsviruslast. LY-CoV555 erzielte den größten Abfall in der mittleren Dosisgruppe (2800 mg) und nicht etwa in der Hochdosisgruppe (7000 mg, MD $>$ LD > HD). In mittlerweile publizierten Endergebnissen von Gottlieb et al. [3] konnte dieser dosisunabhängige Abfall der Viruslast nicht bestätigt werden. Hier führte lediglich die Kombination von LY-CoV555 mit einem weiteren NAb (LY-CoV016: Etesevimab) zur signifikanten Senkung der SARS-CoV-2-Viruslast. REGN-COV2 senkte die SARS-CoV-2-Viruslast am stärksten bei hohen Ausgangsviruslasten sowie Antikörper-negativen Patienten.

In der klinischen Endpunktanalyse (Verum vs. Placebo) beider Studien traten Hospitalisierungen bis Tag 29 (LYCoV555: 1,6\% vs. 6,3\%) bzw. medizinische Behandlungen (REGN-COV2: $3 \%$ vs. $6 \%$ ) in der Verumgruppe seltener auf. Insbesondere bei Risikopatienten mit $\mathrm{BMI}>35 \mathrm{~kg} / \mathrm{m}^{2}$ oder Alter $>65$ Jahre konnte die frühzeitige Gabe von LYCoV555 im Vergleich zu Placebo Hospitalisierungen verhindern ( $4 \%$ vs. $15 \%$ ). Außerdem kam es in der Studienpopulation von Chen et al. lediglich im Placeboarm zu einer Patientenverlegung auf Intensivstation $(0,7 \%$ [1/143] vs. $0 \%$ [0/309] im Verumarm). Es ist davon auszugehen, dass so auch das Auftreten schwerer Verläufe minimiert werden könnte. Insgesamt konnten LYCoV555 und REGN-COV2 bei Auftreten von lediglich $1,6 \%$ bzw. $4,5 \%$ schweren Nebenwirkungen als sicher bewertet werden.

Zusammengefasst konnten die frühzeitig intravenös verabreichten neutra- lisierenden Antikörperkonzentrate LYCoV555 und REGN-COV2 im Vergleich zur Placebogruppe eine stärkere Senkung der SARS-CoV-2-Viruslast im Nasenrachenraum bewirken sowie Hospitalisierungen bzw. Arztkontakte ambulanter COVID-19-Patienten verringern. $\mathrm{Ob}$ die virologische Clearance der Ausgangsviruslast auch direkte klinische „benefits“ in Bezug auf COVID19-assoziierte Symptome hat, könnte in weiterführenden Studien unter Zuhilfenahme der WHO Clinical Progression Scale [2] untersucht werden.

Die Gabe zweier verschiedener NAb scheint zu einer stärkeren Senkung der Ausgangsviruslast zu führen als die Gabe eines einzelnen NAb, da möglicherweise die Gefahr des humoralen Neutralisationsverlusts nach eventuell auftretenden Escape-Mutationen minimiert wird. Aufgrund des Zelleintritts von SARSCoV-2 über den vor allem im NasenRachen-Raum sowie im pulmonalen Endothel vorkommenden Angiotensinconverting-enzyme-2(ACE2)-Rezeptor [4] stellt sich die Frage, ob die intravenöse Verabreichung neutralisierender Antikörper zu einer ausreichenden Anflutung in jenen Kompartimenten führt oder ob eine beispielsweise inhalative Applikationsform zielführender wäre.

Bislang wurde die Wirksamkeit von SARS-CoV-2-neutralisierenden Antikörpern lediglich im Hinblick auf die akute COVID-19 untersucht. Bei vermehrten Hinweisen auf mögliche $\mathrm{Zu}$ sammenhänge von strukturellen [5] und immunologischen [6] Gefäßveränderungen mit dem Auftreten relevanter COVID-19-assoziierter Langzeitfolgen (Post-COVID-Syndrom [PCS]) sollte in weiterführenden Studien untersucht werden, ob die frühzeitige Verabreichung von intravenösen neutralisierenden Antikörpern klinische Auswirkungen auf das weite Spektrum des PCS haben kann.

\section{Korrespondenzadresse}

\section{Dr. med. M. Augustin}

Klinik I für Innere Medizin, Universitätsklinikum Köln (AöR)

Kerpener Str. 62, 50937 Köln, Deutschland

max.augustin@uk-koeln.de

\section{Einhaltung ethischer Richtlinien}

Interessenkonflikt. M. Augustin, M. Hallek und S. Nitschmann geben an, dass kein Interessenkonflikt besteht.

Für diesen Beitrag wurden von den Autoren keine Studien an Menschen oder Tieren durchgeführt. Für die aufgeführten Studien gelten die jeweils dort angegebenen ethischen Richtlinien.

\section{Literatur}

1. Augustin M, Hallek M, Nitschmann S (2020) Remdesivir bei Patienten mit COVID-19. Internist 61:869-872. https://doi.org/10.1007/s00108020-00836-7

2. Characterisation, W.H.O.W.G.o.t.C. and C.-i. Management of (2020) A minimal common outcome measure set for COVID-19 clinical research. Lancet Infect Dis 20(8):e192-e197

3. Gottlieb RL et al (2021) Effect of bamlanivimab as monotherapy or in combination with etesevimab on viral load in patients with mild to moderate COVID-19: a randomized clinical trial. JAMA 325(7):632-644

4. Harrison AG, Lin T, Wang P (2020) Mechanisms of SARS-CoV-2 transmission and pathogenesis. Trends Immunol 41(12):1100-1115

5. Ackermann M et al (2020) Pulmonary vascular endothelialitis, thrombosis, and angiogenesis in Covid-19. NEngl JMed 383(2):120-128

6. Nauen DW et al (2021) Assessing Brain Capillaries in Coronavirus Disease 2019. JAMA Neurol. https:// doi.org/10.1001/jamaneurol.2021.0225 
Hier steht eine Anzeige.

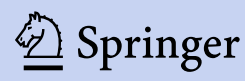

